\title{
Rate Enhancements in the Acetylation and Benzoylation of Certain Aromatic Compounds with Vilsmeier-Haack Reagents Using Acetamide, Benzamide and Oxychlorides under Non-Conventional Conditions
}

\author{
Marri Venkateswarlu, Kamatala Chinna Rajanna*, Mukka Satish Kumar, \\ Utkoor Umesh Kumar, Soma Ramgopal, Pondichery Kuppuswamy Saiprakash \\ Department of Chemistry, Osmania University, Hyderabad, India \\ E-mail: kcrajannaou@yahoo.com \\ Received August 8, 2011; revised September 21, 2011; accepted October 3, 2011
}

\begin{abstract}
Acetylation and benzoylation reactions of certain aromatic aldehydes, ketones with Vilsmeier-Haack Reagents using Acetamide and Oxychloride $\left(\mathrm{SOCl}_{2}\right.$ or $\left.\mathrm{POCl}_{3}\right)$ under conventional (thermal) and non conventional [microwave irradiated (MIR), ultrasonic assisted and solvent free mortar pestle (grinding)] conditions. Reactions afforded good to excellent yields of products with both the $\mathrm{VH}$ reagents, reaction times were fairly less in the case of [amide/ $\mathrm{POCl}_{3}$ ] than those of [amide/ $\left.\mathrm{SOCl}_{2}\right]$ reagent. Reactions are dramatically accelerated in under sonicated and microwave irradiations with a trend: MIR (few seconds) $>>$ Sonication (minutes) $>$ Grinding $(\min )>>$ thermal (several hrs).
\end{abstract}

Keywords: Acetylation, Benzoylation, Vilsmeier-Haack Reagent (VHR), Acetamide, Benzamide, Micro Wave Irradiation, Grinding, Sonication

\section{Introduction}

Acetylation (or ethanoylation in IUPAC nomenclature) describes a reaction that introduces an acetyl functional group into a chemical compound, while benzoylation introduces a benzoyl group into an organic compound. These reactions are included in the category of the most important transformations in Organic Synthesis [1-3]. A reaction involving the replacement of the hydrogen atom of a hydroxyl group with an acetyl group $\left(\mathrm{CH}_{3} \mathrm{CO}\right)$ yields a specific ester, the acetate. Acetylation is one of the principal metabolic pathways of the sulfonamides. It is also one of the important synthetic bio-transformations which operate in the metabolism of drugs in which metabolites are produced that are more readily excreted than the parent drug. However, dogs are exceptional amongst the domesticated species in that acetylation does not occur in their tissues. Acetylation of the $N$-terminal alphaamine of proteins is a widespread modification in eukaryotes. Forty to fifty percent of yeast proteins and between $80 \%$ to $90 \%$ of human proteins are modified in this manner. The pattern of modification is found to be the same throughout evolution.
Among the various protecting groups used for the hydroxyl group, acetyl is one of the most common groups, being stable in the acid reaction conditions and also eases of removal by mild alkaline hydrolysis $[1,2]$. The most commonly used reagent combination for this reaction uses acid anhydride in the presence of acid or base catalysts [3]. Various metal salts [4-16] such as $\mathrm{CoCl}_{2}, \mathrm{TiCl}_{4^{-}}$ $\mathrm{AgClO}_{4}, \mathrm{TaCl}_{5}, \mathrm{TaCl}_{5}-\mathrm{SiO}_{2}, \mathrm{Ce}(\mathrm{III})$ triflate, $\mathrm{Sn}(\mathrm{IV})$ porphyrine and some metal triflates [17-21] such as Sc $(\mathrm{OTf})_{3}, \mathrm{MeSiOTf}, \operatorname{In}(\mathrm{OTf})_{3}, \mathrm{Cu}(\mathrm{OTf})_{2}$ and $\mathrm{Bi}(\mathrm{OTf})_{3}$, bis(cyclopentadienyl) zirconium dichloride [22], $\mathrm{I}_{2}$ [23], 1,3-dibromo-5,5-dimethylhydentoin or trichloroisocyanuric acid [24] have been investigated to meet the demand for more efficient and selective methods.

Benzoylation reaction is generally carried out using benzoyl chloride with a Lewis acid as the benzoylating agent. In addition to benzoyl chloride [1-2], a number of reagents [25-29] such as, benzoic anhydride, benzoyltetrazole, 2-benzoyl-1-methylpyridinium chloride, S-benzoic-O,O-diethylphosphoro-dithoic anhydride, benzoyl cyanide, can be used for carrying out this reaction. Though benzoyl chloride may be a health hazard due to its toxicity, nevertheless it is widely used because of its ready 
availability and low cost. The reaction is usually catalyzed by bases like pyridine, triethylamine and sodium hydroxide [30-31]. Recently Satya Paul et al. [32] developed a rapid, economic and environmentally friendly method for benzoylation of $-\mathrm{NH}_{2},-\mathrm{OH}$ and $-\mathrm{SH}$ groups using $\mathrm{PhCOCl}-\mathrm{Py} /$ basic alumina. The developed reagent system was found to a good alternative to classical method since the benzoylation underwent expeditiously with high yields under solvent-free conditions.

Vilsmeier-Haack reagent ( $\mathrm{VH})$ is one of the most efficient synthetic organic reagents for formylation and acetylation reactions. VH reagents could be prepared form equimolar mixture of formamide or $N, N^{\prime}$-dialkyl formamides such as $N, N^{\prime}$-dimethyl formamide (DMF), $N$, $N$ '-diethyl formamide (DEF), along with oxy halides such as $\mathrm{POCl}_{3}$ and $\mathrm{SOCl}_{2}$ at freezing temperatures. Recent reports on Vilsmeier-Haack $(\mathrm{VH})$ reactions revealed that organic compounds in general and hydrocarbons with excess pi-electrons in particular undergo formylation very easily on synthetic scale [33-49]. However, these reactions are known to afford acetyl derivatives when $N$, $N^{\prime}$-dialkyl formamides are replaced by acetamide or $N$, $N^{\prime}$-dialkyl acetamides such as $N, N^{\prime}$-dimethyl acetamide (DMA) and $N, N^{\prime}$-diethyl acetamide (DEA) in VH reagent. However, not many systematic reports are published in this direction. In view of this the author has embarked on a systematic synthetic study of certain VH acetylation and benzoylation reactions using (Acetamide $\left.+\mathrm{SOCl}_{2}\right) /\left(\right.$ Acetamide $\left.+\mathrm{POCl}_{3}\right)$ or $\left(\right.$ Benzamide $\left.+\mathrm{SOCl}_{2}\right) /$ $\left(\right.$ Benzamide $\left.+\mathrm{POCl}_{3}\right)$ as $\mathrm{VH}$ regents. A set of organic compounds such as aldehydes and ketones are used in these studies, which have been found their importance in a number of industrially important and biologically important reactions.

\section{Experinental Details}

\subsection{General Procedure for Preparation of Vilsmeier-Haack Reagent}

The Vilsmeier Haack (VH) adduct is prepared afresh before use from $\mathrm{SOCl}_{2}$ and acetamide (ACTAM). To a chilled (at $-50^{\circ} \mathrm{C}$ ) actamide (ACTAM) in dichloro ethane (DCE) or acetonitrile (ACN), calculated amount of PO$\mathrm{Cl}_{3}$ was slowly added drop wise to get $\mathrm{VH}$ reagent and stored under cold conditions. Similar procedure is adopted for the preparation of $\mathrm{VH}$ reagent with Benzamide (BNZAM).

\subsection{General Procedure for Vilsmeier-Haack Synthesis in Reflux Condition}

A centimolar $(0.01 \mathrm{~mol})$ organic substrate (aromatic al- dehydes, ketones), about 0.015 moles of $\mathrm{VH}$ reagent and solvent DCE were taken in a cleaned in a Round bottom flask and refluxed till the reaction is completed. After completion of the reaction, as confirmed by TLC, the reaction mixture is treated with $5 \%$ sodium thio sulphate solution, followed by the addition of pet ether. The organic layer was separated, dried over $\mathrm{Na}_{2} \mathrm{SO}_{4}$ and evaporated under vacuum, purified with column chromatography using DCE: n-hexane (8:2) as eluent to get pure product. This methodology has also been successfully used for ACN mediated reactions. This methodology has been successfully is adopted for CAN mediated reactions. The yields of major products are compiled in Tables 1 to 4 .

\subsection{General Procedure for Vilsmeier-Haack Synthesis under Solvent Free Conditions by Grinding in Mortar with Pestle}

A centimolar $(0.01 \mathrm{~mol})$ organic substrate (aldehydes, ketones or acetophenone), and about 0.015 moles of $\mathrm{VH}$ reagent were taken in a previously cleaned mortar and grounded till the reaction is completed. After completion of the reaction, as checked by TLC, the reaction mixture is treated with $5 \%$ sodium thio sulphate solution, followed by the addition of pet ether. The organic layer was separated, dried over $\mathrm{Na}_{2} \mathrm{SO}_{4}$ and evaporated under vacuum, purified with column chromatography using DCE: $\mathrm{n}$-hexane (8:2) as eluent to get pure product. The yields of major products are given in Tables 1 to 4 .

\subsection{General Procedure for Sonicated Vilsmeier-Haack Synthesis}

A centimolar $(0.01 \mathrm{~mol})$ organic substrate (aldehydes, ketones or acetophenone), about 0.015 moles of $\mathrm{VH}$ reagent and solvent DCE were taken in a cleaned in a Round bottom flask and clamped in a sonicator and progress of the reaction is monitored by TLC. After completion of the reaction, as ascertained by TLC, the reaction mixture is treated with $5 \%$ sodium thio sulphate solution, followed by a the same work-up procedure as mentioned the above section to get the final product. This methodology has also been successfully for ACN mediated reactions. The yields of major products are shown in Tables 1 to 4 .

\subsection{General Procedure for MW Irradiated Vilsmeier-Haack Synthesis}

Methodology adopted for MW assisted VH synthesis is almost similar to that used in the above section. A centimolar $(0.01 \mathrm{~mol})$ organic substrate (aldehydes, ketones or acetophenone), about 0.015 moles of $\mathrm{VH}$ reagent and 
Table 1. VH acetylation reactions with (Acetamide + $\mathrm{SOCl}_{2}$ ) and carbonyl compounds.

\begin{tabular}{|c|c|c|c|c|c|c|c|c|c|}
\hline \multirow{2}{*}{ Entry } & \multirow{2}{*}{ Substrate } & \multicolumn{2}{|c|}{ Thermal (Room temp) } & \multicolumn{2}{|c|}{ Grinding (Solvent free) } & \multicolumn{2}{|c|}{ Sonication } & \multicolumn{2}{|c|}{ Microwave (300 watt) } \\
\hline & & RT (hrs) & Yield (\%) & $\mathrm{RT}$ (min) & Yield (\%) & $\mathrm{RT}(\min )$ & Yield (\%) & $\mathrm{RT}(\mathrm{sec})$ & Yield (\%) \\
\hline 1 & Benzaldehyde & 13 & 68 & 120 & 70 & 90 & 65 & 200 & 70 \\
\hline 2 & Salicyl adehyde & 13 & 70 & 140 & 75 & 90 & 78 & 200 & 75 \\
\hline 3 & 4-OH benzaldehyde & 11 & 80 & 110 & 83 & 90 & 79 & 180 & 80 \\
\hline 4 & 4-OMe benzaldehyde & 12 & 64 & 110 & 63 & 90 & 67 & 200 & 68 \\
\hline 5 & 4-Cl benzaldehyde & 11 & 67 & 100 & 63 & 90 & 67 & 200 & 65 \\
\hline 6 & 4-Br benzaldehyde & 12 & 7 & 110 & 69 & 90 & 63 & 200 & 77 \\
\hline 8 & Cinamaldehyde & 13 & 69 & 130 & 70 & 90 & 69 & 200 & 73 \\
\hline 9 & Acetophenone & 12 & 76 & 110 & 73 & 90 & 80 & 190 & 77 \\
\hline 10 & 2-OH acetophenone & 11 & 78 & 110 & 80 & 90 & 76 & 190 & 81 \\
\hline 11 & 4-OH acetophenone & 11 & 69 & 110 & 74 & 90 & 71 & 200 & 68 \\
\hline 12 & 4-Me acetophenone & 10 & 64 & 140 & 61 & 90 & 66 & 200 & 65 \\
\hline 13 & 3-OH acetophenone & 11 & 69 & 110 & 73 & 90 & 71 & 200 & 71 \\
\hline 14 & 4-Br acetophenone & 13 & 65 & 110 & 69 & 90 & 66 & 200 & 64 \\
\hline 15 & 4- $\mathrm{NO}_{2}$ acetophenone & 12 & 85 & 100 & 80 & 90 & 79 & 180 & 81 \\
\hline
\end{tabular}

Table 2. VH acetylation reactions with (Acetamide $+\mathbf{P O C l}_{3}$ ) and carbonyl compounds.

\begin{tabular}{|c|c|c|c|c|c|c|c|c|c|}
\hline \multirow{2}{*}{ Entry } & \multirow{2}{*}{ Substrate } & \multicolumn{2}{|c|}{ Thermal (Room temp) } & \multicolumn{2}{|c|}{ Grinding (Solvent free) } & \multicolumn{2}{|c|}{ Sonication } & \multicolumn{2}{|c|}{ Microwave (300 watt) } \\
\hline & & RT (hrs) & Yield (\%) & $\mathrm{RT}(\min )$ & Yield (\%) & $\mathrm{RT}(\min )$ & Yield (\%) & $\mathrm{RT}(\mathrm{sec})$ & Yield (\%) \\
\hline 1 & Benzaldehyde & 11 & 69 & 120 & 70 & 90 & 65 & 200 & 70 \\
\hline 2 & Salicyladehyde & 12 & 70 & 140 & 75 & 90 & 78 & 200 & 75 \\
\hline 3 & 4-OH benzaldehyde & 11 & 83 & 110 & 80 & 90 & 80 & 180 & 79 \\
\hline 4 & 4-OMe benzaldehyde & 12 & 67 & 110 & 68 & 90 & 67 & 200 & 68 \\
\hline 5 & 4-Cl benzaldehyde & 11 & 70 & 100 & 69 & 90 & 70 & 200 & 67 \\
\hline 6 & 4-Br benzaldehyde & 12 & 72 & 110 & 66 & 90 & 63 & 200 & 77 \\
\hline 7 & 4- $\mathrm{NO}_{2}$ benzaldehyde & 11 & 79 & 110 & 82 & 90 & 78 & 190 & 82 \\
\hline 9 & Acetophenone & 12 & 77 & 110 & 74 & 90 & 77 & 190 & 80 \\
\hline 10 & 2-OH acetophenone & 11 & 76 & 110 & 80 & 90 & 69 & 190 & 81 \\
\hline 11 & 4-OH acetophenone & 11 & 74 & 110 & 75 & 90 & 71 & 200 & 80 \\
\hline 12 & 4-Me acetophenone & 12 & 66 & 140 & 65 & 90 & 66 & 200 & 69 \\
\hline 13 & 3-OH acetophenone & 11 & 68 & 110 & 73 & 90 & 77 & 200 & 71 \\
\hline 14 & 4-Br acetophenone & 13 & 64 & 110 & 69 & 90 & 68 & 200 & 64 \\
\hline 15 & 4- $\mathrm{NO}_{2}$ acetophenone & 12 & 80 & 100 & 84 & 90 & 79 & 180 & 85 \\
\hline
\end{tabular}


Table 3. VH benzoylation reactions with (Benzamide $+\mathrm{SOCl}_{2}$ ) and carbonyl compounds.

\begin{tabular}{|c|c|c|c|c|c|c|c|c|c|}
\hline \multirow{2}{*}{ Entry } & \multirow{2}{*}{ Substrate } & \multicolumn{2}{|c|}{ Thermal (Room temp) } & \multicolumn{2}{|c|}{ Grinding (Solvent free) } & \multicolumn{2}{|c|}{ Sonication } & \multicolumn{2}{|c|}{ Microwave (300 watt) } \\
\hline & & RT (hrs) & Yield (\%) & RT (min) & (Yield) (\%) & $\mathrm{RT}(\min )$ & Yield (\%) & $\mathrm{RT}(\mathrm{sec})$ & Yield (\%) \\
\hline 1 & Benzaldehyde & 12 & 65 & 120 & 67 & 90 & 62 & 200 & 69 \\
\hline 2 & Salicyladehyde & 12 & 70 & 110 & 75 & 90 & 76 & 200 & 72 \\
\hline 3 & 4-OH benzaldehyde & 11 & 80 & 110 & 81 & 90 & 79 & 190 & 76 \\
\hline 4 & 4-OMe benzaldehyde & 13 & 67 & 130 & 63 & 90 & 66 & 200 & 68 \\
\hline 5 & 4-Cl benzaldehyde & 13 & 63 & 130 & 67 & 90 & 65 & 220 & 62 \\
\hline 6 & 4-Br benzaldehyde & 12 & 70 & 130 & 68 & 90 & 63 & 200 & 72 \\
\hline 7 & 4- $\mathrm{NO}_{2}$ benzaldehyde & 11 & 81 & 100 & 80 & 90 & 75 & 180 & 82 \\
\hline 8 & Cinamaldehyde & 13 & 62 & 110 & 70 & 90 & 67 & 200 & 71 \\
\hline 9 & Acetophenone & 12 & 74 & 110 & 76 & 90 & 80 & 190 & 79 \\
\hline 10 & 2-OH acetophenone & 12 & 80 & 110 & 78 & 90 & 81 & 190 & 76 \\
\hline 11 & 4-OH acetophenone & 12 & 71 & 110 & 74 & 90 & 69 & 200 & 72 \\
\hline 12 & 4-Me acetophenone & 11 & 64 & 140 & 61 & 90 & 66 & 200 & 65 \\
\hline 13 & 3-OH acetophenone & 11 & 69 & 110 & 73 & 90 & 71 & 200 & 68 \\
\hline 14 & 4-Br acetophenone & 11 & 68 & 110 & 65 & 90 & 63 & 200 & 64 \\
\hline 15 & 4- $\mathrm{NO}_{2}$ acetophenone & 10 & 85 & 100 & 80 & 90 & 79 & 180 & 81 \\
\hline
\end{tabular}

Table 4. VH benzoylation reactions with (Benzamide $+\mathbf{P O C l}_{3}$ ) and carbonyl compounds.

\begin{tabular}{|c|c|c|c|c|c|c|c|c|c|}
\hline \multirow{2}{*}{ Entry } & \multirow{2}{*}{ Substrate } & \multicolumn{2}{|c|}{ Thermal (Room temp) } & \multicolumn{2}{|c|}{ Grinding (Solvent free) } & \multicolumn{2}{|c|}{ Sonication } & \multicolumn{2}{|c|}{ Microwave (300 watt) } \\
\hline & & RT (hrs) & Yield (\%) & $\mathrm{RT}$ (min) & (Yield) (\%) & $\mathrm{RT}(\min )$ & Yield (\%) & $\mathrm{RT}(\mathrm{sec})$ & Yield (\%) \\
\hline 1 & Benzaldehyde & 11 & 69 & 120 & 70 & 90 & 65 & 200 & 70 \\
\hline 2 & Salicyladehyde & 12 & 70 & 140 & 75 & 90 & 78 & 200 & 75 \\
\hline 3 & 4-OH benzaldehyde & 11 & 83 & 110 & 80 & 90 & 80 & 180 & 79 \\
\hline 4 & 4-OMe benzaldehyde & 12 & 67 & 110 & 68 & 90 & 67 & 200 & 68 \\
\hline 5 & 4-Cl benzaldehyde & 11 & 75 & 100 & 69 & 90 & 76 & 200 & 74 \\
\hline 6 & 4-Br benzaldehyde & 12 & 76 & 110 & 67 & 90 & 65 & 200 & 77 \\
\hline 7 & 4- $\mathrm{NO}_{2}$ benzaldehyde & 11 & 79 & 110 & 82 & 90 & 80 & 190 & 82 \\
\hline 8 & Cinamaldehyde & 13 & 73 & 130 & 71 & 90 & 73 & 200 & 75 \\
\hline 9 & Acetophenone & 12 & 77 & 110 & 79 & 90 & 77 & 190 & 80 \\
\hline 10 & 2-OH acetophenone & 11 & 76 & 110 & 80 & 90 & 69 & 190 & 81 \\
\hline 11 & 4-OH acetophenone & 11 & 75 & 110 & 77 & 90 & 73 & 200 & 80 \\
\hline 12 & 4-Me acetophenone & 12 & 67 & 140 & 65 & 90 & 68 & 200 & 69 \\
\hline 13 & 3-OH acetophenone & 11 & 70 & 110 & 74 & 90 & 77 & 200 & 73 \\
\hline 14 & 4-Br acetophenone & 13 & 69 & 110 & 70 & 90 & 68 & 200 & 67 \\
\hline 15 & 4- $\mathrm{NO}_{2}$ acetophenone & 12 & 81 & 100 & 84 & 90 & 79 & 180 & 84 \\
\hline
\end{tabular}


solvent DCE were taken in a cleaned in a Round bottom flask and clamped in a laboratory MW oven. Progress of the reaction, under micro wave irradiated conditions, is followed by TLC. After completion of the reaction, as ascertained by TLC, the reaction mixture is treated with $5 \%$ sodium thio sulphate solution, followed by a the same work-up procedure as mentioned the above section to get the final product. This methodology has also been successfully extended for ACN mediated reactions. The yields of major products are presented in Tables $\mathbf{1}$ to $\mathbf{4}$.

\subsection{Product Analysis}

TLC pure products were characterized by spectroscopic methods. The melting points were determined on Mettler FP 51 (Neo Pharma Instrument Corp.) and are not corrected. We have recorded $\mathrm{H}$ NMR spectra on a Jeol FX$90 \mathrm{~A}$ instrument in chloroform-d using TMS as internal standard. The mass spectra were recorded on a VG micromass $70-70 \mathrm{H}$ spectrometer. The IR was recorded on a Nicolet 740 FT-IR spectrometer. The UV was recorded on Shimadzu-240 UV-Visible spectrophotometer. Spectroscopic data for certain representative isolated products compiled Tables 5 and $\mathbf{6}$.

\section{Results and Discussion}

Aromatic compounds such as Benzaldehyde and Acetophenone derivatives underwent acetylation under Vilsmeier-Haack conditions in fairly good to very good yields when treated with [Acetamide/ $\mathrm{SOCl}_{2}$ ] and [Acetamide/ $\mathrm{POCl}_{3}$ ] respectively under conventional and nonconventional conditions. However, to check the generality of the reaction an array of substituted aromatic aldehydes and ketones are used as substrates under present reaction conditions as shown in Scheme 1. The yields of major products are compiled in Tables 1 to 4 . The products were characterized by ${ }^{1} \mathrm{H}-\mathrm{NMR}$, Mass spectra with authentic samples and found to be satisfactory. It is of interest to note that orthohydroxy acetophenone (OHAP) underwent cyclization followed by acetylation and afforded acetyl chromone as described in earlier workers.
However, Meta (MHAP) and para hydroxy acetophenones (PHAP) did not undergo cyclization but afforded acetyl derivatives. The difference in the reactivity of OHAP from MHAP and PHAP could be attributed to the fact that the -OH group is away from the carbonyl (main) functional group which is favorable to form a stable ring through cyclization.

Data for VH synthesis of acetylation/benzoylation reactions are presented in tables 1 to 4 and Figures 1 and 2, which clearly indicate remarkable rate enhancements and increase in reaction yields from thermal reactions to microwave assisted reactions with an increasing trend: MIR >> Sonication >> Grinding (mortar-pestle) > thermal. Reactions are too sluggish under times in thermal conditions even at elevated temperatures. The reaction times are in the range of 10 to 12 hours. However, the reactions are completed only in 100 - 120 min under solvent free conditions suggesting that the present method could be employed for small scale. Progress of the reaction under solvent free conditions might be due to the heat energy generated from the mechanical energy when the reactionmixture is grounded in the mortar. The yields are highly superior over corresponding solution phase reactions. Since the reaction time of solvent free reaction is at least six times less than corresponding solution phase reaction and avoids the use of toxic solvents, this methodology could be considered as a green chemistry oriented synthesis of $\mathrm{VH}$ reactions. Rate enhancements in the case of grinding reactions could be probably attributed to the faster activation of molecules due to the direction friction followed by conversion of mechanical energy into heat energy. In the case of sonicated reactions reaction times are further reduced to 90 minutes. The rate accelerations under sonicated could be explained by the cavitation phenomenon. Activation of molecules is done by the in situ energy released due to the rupture of cavitation bubbles generated by ultrasonic waves. It is also of interest to note that the reactions times are reduced from several minutes to only few seconds under microwave irradiation (MIR). The extremely faster reaction rates in MIR system could be due to the bulk activation of molecules rather than random activation.
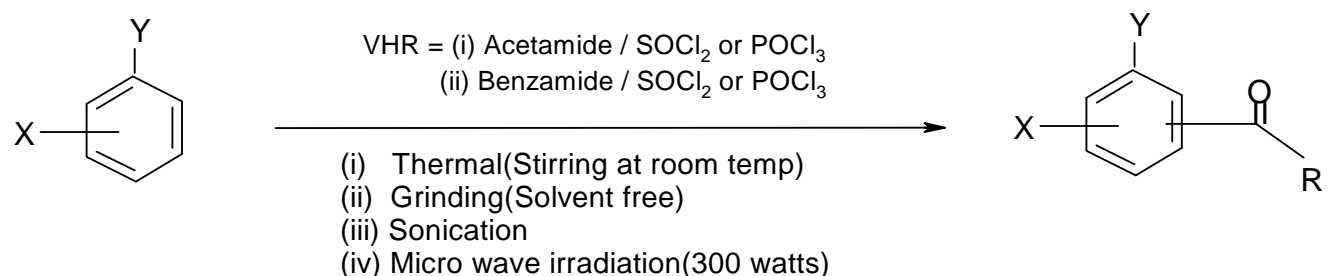

(iv) Micro wave irradiation(300 watts)

Where $\mathrm{R}=\mathrm{CH}_{3}$ when $\mathrm{VHR}=\left(\right.$ Acetamide $\left.+\mathrm{SOCl}_{2}\right)$ or $\left(\right.$ Acetamide $\left.+\mathrm{POCl}_{3}\right) ; \mathrm{R}=\mathrm{C}_{6} \mathrm{H}_{5}$ when $\mathrm{VHR}=(\mathrm{Benzamide}+\mathrm{SOCl})_{2}$ or $(\mathrm{Benzamide}+\mathrm{POCl}) ; \mathrm{Y}=\mathrm{CHO}_{3}$ $\mathrm{COCH}_{3} ; \mathrm{X}=$ electron donating or electron withdrawing groups.

Scheme 1. Acetylation and benzoylation of benzaldehydes and acetophenones under Vilsmeier Haack conditions. 
Table 5. Spectroscopic data for VH acetylation of aromatic carbonyl compounds.

\begin{tabular}{|c|c|c|c|c|}
\hline \multirow{2}{*}{ Entry } & \multirow{2}{*}{ Substrate } & \multirow{2}{*}{ Product } & \multicolumn{2}{|r|}{ Spectroscopic data } \\
\hline & & & $\mathrm{m} / \mathrm{z}$ & ${ }^{1} \mathrm{H}$ NMR \\
\hline 1 & Benzaldehyde & 3-Acetyl Benzaldehyde & 148 & $\begin{array}{c}\delta 2.65\left(\mathrm{~s} 3 \mathrm{H}, \mathrm{CH}_{3}\right) ; \delta 7.66(\mathrm{~m} 1 \mathrm{H}, \mathrm{Ar}) ; \delta 8.2(\mathrm{~d} 2 \mathrm{H}, \mathrm{Ar}) \\
\delta 8.5(\mathrm{~s} 1 \mathrm{H}, \mathrm{Ar}) ; \delta 9.9(\mathrm{~s} 1 \mathrm{H}, \mathrm{Ar}-\mathrm{CHO})\end{array}$ \\
\hline 2 & Salicyladehyde & 3-Acetyl Salicyladehyde & 164 & $\begin{array}{c}\delta 2.65\left(\mathrm{~s} 3 \mathrm{H}, \mathrm{CH}_{3}\right) ; \delta 7.2(\mathrm{~m} 1 \mathrm{H}, \mathrm{Ar}) ; \delta 7.6(\mathrm{~d} 1 \mathrm{H}, \mathrm{Ar}) \\
\delta 7.9(\mathrm{~d} 1 \mathrm{H}, \mathrm{Ar}) ; \delta 10.3(\mathrm{~s} 1 \mathrm{H}, \mathrm{Ar}-\mathrm{CHO}) ; \delta 11.05(\mathrm{~s} 1 \mathrm{H}, \mathrm{Ar}-\mathrm{OH})\end{array}$ \\
\hline 3 & 4-OH benzaldehyde & 3-Acetyl 4-OH benzaldehyde & 164 & $\begin{array}{c}\delta 2.65\left(\mathrm{~s} 3 \mathrm{H}, \mathrm{CH}_{3}\right) ; \delta 7.1(\mathrm{~d} 1 \mathrm{H}, \mathrm{Ar}) ; \delta 7.95(\mathrm{~d} 1 \mathrm{H}, \mathrm{Ar}) \\
\delta 8.3(\mathrm{~s} 1 \mathrm{H}, \mathrm{Ar}) ; \delta 9.5(\mathrm{~s} 1 \mathrm{H}, \mathrm{Ar}-\mathrm{CHO}) ; \delta 10.5(\mathrm{~s} 1 \mathrm{H}, \mathrm{Ar}-\mathrm{OH})\end{array}$ \\
\hline 4 & 4-OMe benzaldehyde & 3-Acetyl 4-OMe benzaldehyde & 178 & $\begin{array}{l}\delta 2.65\left(\mathrm{~s} 3 \mathrm{H}, \mathrm{CH}_{3}\right) ; \delta 3.93\left(\mathrm{~s} 3 \mathrm{H}, \mathrm{Ar}-\mathrm{OCH}_{3}\right) ; \delta 7.15(\mathrm{~d} \mathrm{1H}, \mathrm{Ar}) \\
\quad \delta 8.0(\mathrm{~d} 1 \mathrm{H}, \mathrm{Ar}) ; \delta 8.4(\mathrm{~s} 1 \mathrm{H}, \mathrm{Ar}) ; \delta 10.05(\mathrm{~s} 1 \mathrm{H}, \mathrm{Ar}-\mathrm{CHO})\end{array}$ \\
\hline 5 & 4-Cl benzaldehyde & 3-Acetyl 4-Cl benzaldehyde & 182 & $\begin{array}{c}\delta 2.65\left(\mathrm{~s} 3 \mathrm{H}, \mathrm{CH}_{3}\right) ; \delta 7.65(\mathrm{~d} 1 \mathrm{H}, \mathrm{Ar}) ; \delta 8.05(\mathrm{~d} 1 \mathrm{H}, \mathrm{Ar}) \\
\delta 8.4(\mathrm{~s} 1 \mathrm{H}, \mathrm{Ar}) ; \delta 9.95(\mathrm{~s} 1 \mathrm{H}, \mathrm{Ar}-\mathrm{CHO})\end{array}$ \\
\hline 6 & 4-Br benzaldehyde & 3-Acetyl 4-Br benzaldehyde & 226 & $\begin{array}{c}\delta 2.63\left(\mathrm{~s} 3 \mathrm{H}, \mathrm{CH}_{3}\right) ; \delta 7.78(\mathrm{~d} 1 \mathrm{H}, \mathrm{Ar}) ; \delta 7.95(\mathrm{~d} 1 \mathrm{H}, \mathrm{Ar}) \\
\delta 8.5(\mathrm{~s} 1 \mathrm{H}, \mathrm{Ar}) ; \delta 10.0(\mathrm{~s} 1 \mathrm{H}, \mathrm{Ar}-\mathrm{CHO})\end{array}$ \\
\hline 7 & 4- $\mathrm{NO}_{2}$ benzaldehyde & 3-Acetyl 4- $\mathrm{NO}_{2}$ benzaldehyde & 193 & $\begin{array}{c}\delta 2.66\left(\mathrm{~s} 3 \mathrm{H}, \mathrm{CH}_{3}\right) ; \delta 8.35(\mathrm{~d} 1 \mathrm{H}, \mathrm{Ar}) ; \delta 8.55(\mathrm{~d} 1 \mathrm{H}, \mathrm{Ar}) \\
\delta 8.75(\mathrm{~s} 1 \mathrm{H}, \mathrm{Ar}) ; \delta 9.95(\mathrm{~s} 1 \mathrm{H}, \mathrm{Ar}-\mathrm{CHO})\end{array}$ \\
\hline 8 & Cinnamaldehyde & 3-Acetyl Cinnamaldehyde & 174 & $\begin{array}{c}\delta 2.65\left(\mathrm{~s} 3 \mathrm{H}, \mathrm{CH}_{3}\right) ; \delta 6.75(\mathrm{~d} 1 \mathrm{H},=\mathrm{CH}) ; \delta 7.0(\mathrm{~m} \mathrm{1H}, \mathrm{Ar}) \\
\delta 7.53(\mathrm{~d} 1 \mathrm{H}, \mathrm{Ar}) ; \delta 7.68(\mathrm{~s} 1 \mathrm{H}, \mathrm{Ar}-\mathrm{CH}=) ; \delta 7.85(\mathrm{~d} 1 \mathrm{H}, \mathrm{Ar}) \\
\delta 8.05(\mathrm{~s} 1 \mathrm{H}, \mathrm{Ar}) ; \delta 9.9(\mathrm{~s} 1 \mathrm{H}, \mathrm{Ar}-\mathrm{CHO})\end{array}$ \\
\hline 9 & Acetophenone & 3-Acetyl Acetophenone & 162 & $\begin{array}{c}\delta 2.8\left(\mathrm{~s} 6 \mathrm{H}, \mathrm{CH}_{3}\right) ; \delta 6.7(\mathrm{~m} 1 \mathrm{H}, \mathrm{Ar}) ; \delta 8.2(\mathrm{~d} \mathrm{2H}, \mathrm{Ar}) \\
\delta 8.6(\mathrm{~s} 1 \mathrm{H}, \mathrm{Ar})\end{array}$ \\
\hline 10 & 2- $\mathrm{OH}$ acetophenone & 3-Acetyl Chromone & 187 & $\begin{array}{c}\delta 2.6\left(\mathrm{~s} 3 \mathrm{H}, \mathrm{CH}_{3}\right) ; \delta 6.65(\mathrm{~d} 2 \mathrm{H}, \mathrm{Ar}) ; \delta 7.4(\mathrm{~m} \mathrm{2H}, \mathrm{Ar}) \\
\delta 8.2(\mathrm{~s} 1 \mathrm{H}, \mathrm{Ar})\end{array}$ \\
\hline 11 & 4-OH acetophenone & 3-Acetyl 4-OH acetophenone & 178 & $\begin{array}{c}\delta 2.8\left(\mathrm{~s} 6 \mathrm{H}, \mathrm{CH}_{3}\right) ; \delta 7.0(\mathrm{~d} 1 \mathrm{H}, \mathrm{Ar}) ; \delta 8.0(\mathrm{~d} 1 \mathrm{H}, \mathrm{Ar}) \\
\delta 8.6(\mathrm{~d} 1 \mathrm{H}, \mathrm{Ar}) ; \delta 11.4(\mathrm{~d} 1 \mathrm{H}, \mathrm{Ar}-\mathrm{OH})\end{array}$ \\
\hline 13 & 3-OH acetophenone & 3-Acetyl 3-OH acetophenone & 178 & $\begin{array}{c}\delta 2.8\left(\mathrm{~s} 3 \mathrm{H}, \mathrm{CH}_{3}\right) ; \delta 7.45(\mathrm{~s} 1 \mathrm{H}, \mathrm{Ar}) ; \delta 7.6-7.8(\mathrm{~m} 5 \mathrm{H}, \mathrm{Ar}) \\
\delta 7.95(\mathrm{~s} 2 \mathrm{H}, \mathrm{Ar}) ; \delta 10.05(\mathrm{~s} 1 \mathrm{H}, \mathrm{Ar})\end{array}$ \\
\hline 14 & 4-Br acetophenone & 3-Acetyl 4-Br acetophenone & 240 & $\begin{array}{c}\delta 2.8\left(\mathrm{~s} 6 \mathrm{H}, \mathrm{CH}_{3}\right) ; \delta 7.4(\mathrm{~d} 1 \mathrm{H}, \mathrm{Ar}) ; \delta 8.05(\mathrm{~d} 1 \mathrm{H}, \mathrm{Ar}) \\
\delta 8.7(\mathrm{~d} 1 \mathrm{H}, \mathrm{Ar})\end{array}$ \\
\hline 15 & 4- $\mathrm{NO}_{2}$ acetophenone & 3-Acetyl 4- $\mathrm{NO}_{2}$ acetophenone & 207 & $\begin{array}{c}\delta 2.8\left(\mathrm{~s} 6 \mathrm{H}, \mathrm{CH}_{3}\right) ; \delta 7.5(\mathrm{~d} 1 \mathrm{H}, \mathrm{Ar}) ; \delta 8.1(\mathrm{~d} 1 \mathrm{H}, \mathrm{Ar}) \\
\delta 8.8(\mathrm{~d} 1 \mathrm{H}, \mathrm{Ar})\end{array}$ \\
\hline
\end{tabular}

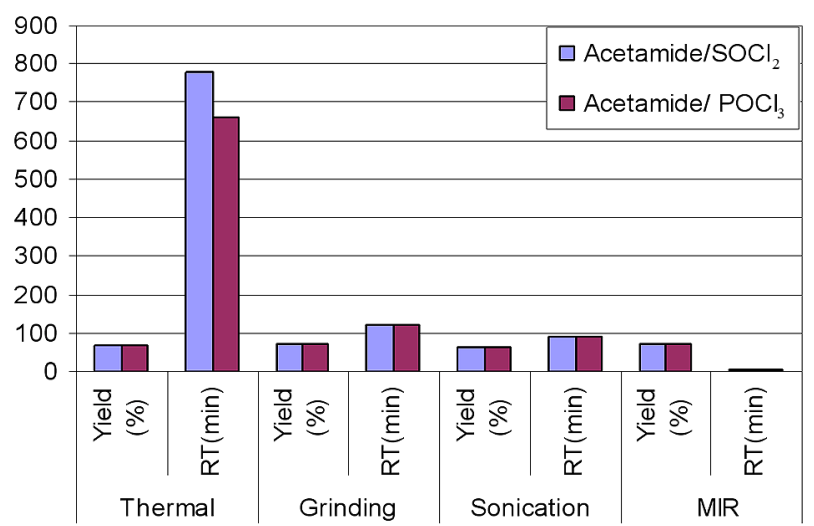

Figure 1. VH acetylation of benzaldehyde under thermal and non-conventional conditions.

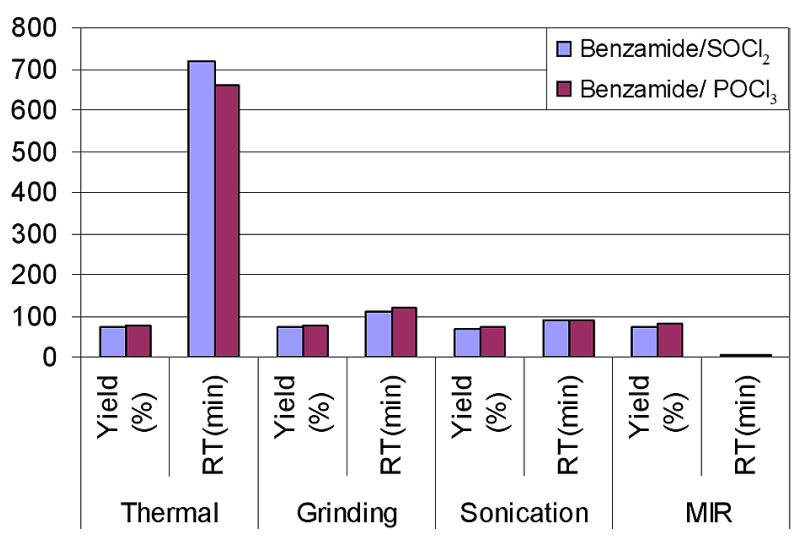

Figure 2. VH benzoylation of 4-OHAP under thermal and non-conventional conditions. 
Table 6. Spectroscopic data for VH benzoylation of aromatic carbonyl compounds.

\begin{tabular}{|c|c|c|c|c|}
\hline \multirow{2}{*}{ Entry } & \multirow{2}{*}{ Substrate } & \multirow{2}{*}{ Product } & \multicolumn{2}{|r|}{ Spectral data } \\
\hline & & & $\mathrm{m} / \mathrm{z}$ & ${ }^{1} \mathrm{H}$ NMR \\
\hline 1 & Benzaldehyde & 3-Benzoyl Benzaldehyde & 210 & $\begin{array}{c}\delta 7.35(\mathrm{~m} 4 \mathrm{H}, \mathrm{Ar}) ; \delta 7.65(\mathrm{~d} 2 \mathrm{H}, \mathrm{Ar}) ; \delta 8.0(\mathrm{~d} 2 \mathrm{H}, \mathrm{Ar}) \\
\delta 8.37(\mathrm{~s} 1 \mathrm{H}, \mathrm{Ar}) ; \delta 10.05(\mathrm{~s} 1 \mathrm{H}, \mathrm{Ar}-\mathrm{CHO})\end{array}$ \\
\hline 2 & Salicyladehyde & 3-Benzoyl Salicyladehyde & 258 & $\delta 7.35-7.92(\mathrm{~m} 8 \mathrm{H}, \mathrm{Ar}) ; \delta 10.1(\mathrm{~s} 1 \mathrm{H} \mathrm{CHO}) ; \delta 10.7(\mathrm{~s} 1 \mathrm{H}, \mathrm{Ar}-\mathrm{OH})$ \\
\hline 3 & 4-OH benzaldehyde & 3-Benzoyl 4-OH benzaldehyde & 258 & $\begin{array}{c}\delta 7.3(\mathrm{~m} 4 \mathrm{H}, \mathrm{Ar}) ; \delta 7.6(\mathrm{~d} 1 \mathrm{H}, \mathrm{Ar}) ; \delta 8.0(\mathrm{~d} 2 \mathrm{H}, \mathrm{Ar}) \\
\delta 8.3(\mathrm{~s} 1 \mathrm{H}, \mathrm{Ar}) ; \delta 9.95(\mathrm{~s} 1 \mathrm{H}, \mathrm{Ar}-\mathrm{CHO}) ; \delta 10.95(\mathrm{~s} 2 \mathrm{H}, \mathrm{Ar}-\mathrm{OH})\end{array}$ \\
\hline 4 & 4-OMe benzaldehyde & 3-Benzoyl 4-OMe benzaldehyde & 240 & $\begin{array}{c}\delta 3.7\left(\mathrm{~s} 3 \mathrm{H}, \mathrm{OCH}_{3}\right) ; \delta 7.4(\mathrm{~m} \mathrm{4H}, \mathrm{Ar}) ; \delta 7.75(\mathrm{~d} \mathrm{1H}, \mathrm{Ar}) \\
\delta 7.9(\mathrm{~d} 2 \mathrm{H}, \mathrm{Ar}) ; \delta 7.75(\mathrm{~d} \mathrm{1H}, \mathrm{Ar}) ; \delta 7.9(\mathrm{~d} 2 \mathrm{H}, \mathrm{Ar}) \\
\delta 8.2(\mathrm{~d} 1 \mathrm{H}, \mathrm{Ar}) ; \delta 10.05(\mathrm{~s} 1 \mathrm{H}, \mathrm{Ar}-\mathrm{CHO})\end{array}$ \\
\hline 5 & 4-Cl benzaldehyde & 3-Benzoyl 4-Cl benzaldehyde & 244 & $\begin{array}{c}\delta 7.4(\mathrm{~m} 4 \mathrm{H}, \mathrm{Ar}) ; \delta 7.7(\mathrm{~d} 1 \mathrm{H}, \mathrm{Ar}) ; \delta 8.3(\mathrm{~d} \mathrm{2H}, \mathrm{Ar}) \\
\delta 8.45(\mathrm{~s} 1 \mathrm{H}, \mathrm{Ar}) ; \delta 10.0(\mathrm{~s} 1 \mathrm{H}, \mathrm{Ar}-\mathrm{CHO})\end{array}$ \\
\hline 6 & 4-Br benzaldehyde & 3-Benzoyl 4-Br benzaldehyde & 288 & $\begin{array}{c}\delta 7.4(\mathrm{~m} 4 \mathrm{H}, \mathrm{Ar}) ; \delta 7.7(\mathrm{~d} 1 \mathrm{H}, \mathrm{Ar}) ; \delta 8.0(\mathrm{~d} 2 \mathrm{H}, \mathrm{Ar}) \\
\delta 8.2(\mathrm{~d} 1 \mathrm{H}, \mathrm{Ar}) ; \delta 9.8(\mathrm{~s} 1 \mathrm{H}, \mathrm{Ar}-\mathrm{CHO})\end{array}$ \\
\hline 7 & 4- $\mathrm{NO}_{2}$ benzaldehyde & 3-Benzoyl 4- $\mathrm{NO}_{2}$ benzaldehyde & 255 & $\begin{array}{c}\delta 7.35(\mathrm{~m}, 4 \mathrm{H} \mathrm{Ar}) ; \delta 7.65(\mathrm{~d}, 1 \mathrm{H}, \mathrm{Ar}) ; \delta 8.05(\mathrm{~d}, 2 \mathrm{H}, \mathrm{Ar}) \\
\delta 8.35(\mathrm{~s} 1 \mathrm{H}, \mathrm{Ar}) ; \delta 9.95(\mathrm{~s} 1 \mathrm{H}, \mathrm{Ar}-\mathrm{CHO})\end{array}$ \\
\hline 8 & Cinnamaldehyde & 3-Benzoyl Cinnamaldehyde & 235 & $\begin{array}{c}\delta 6.75(\mathrm{~d}, 1 \mathrm{H},=\mathrm{CH}) ; \delta 7.4(\mathrm{~m}, 1 \mathrm{H}, \mathrm{Ar}) ; \delta 7.45(\mathrm{~d} 1 \mathrm{H}, \mathrm{Ar}) \\
\delta 7.5-7.8(\mathrm{~m} 7 \mathrm{H}, \mathrm{Ar}) ; \delta 8.05(\mathrm{~d} \mathrm{1H},=\mathrm{CH}) ; \delta 9.9(\mathrm{~s} 1 \mathrm{H}, \mathrm{Ar})\end{array}$ \\
\hline 9 & Acetophenone & 3-Benzoyl Acetophenone & 224 & $\begin{array}{c}\delta 2.8\left(\mathrm{~s} 3 \mathrm{H}, \mathrm{CH}_{3}\right) ; \delta 7.8(\mathrm{~m} \mathrm{5H}, \mathrm{Ar}) ; \delta 7.6(\mathrm{~m} 1 \mathrm{H}, \mathrm{Ar}) \\
\delta 7.95(\mathrm{~d} 2 \mathrm{H}, \mathrm{Ar}) ; \delta 8.45(\mathrm{~s} 1 \mathrm{H}, \mathrm{Ar})\end{array}$ \\
\hline 10 & 2-OH acetophenone & 3-Benzoyl Chromone & 250 & $\begin{aligned} \delta 6.34(\mathrm{~s} 1 \mathrm{H}, \mathrm{Ar}) & ; \delta 7.4(\mathrm{~m} 6 \mathrm{H}, \mathrm{Ar}) ; \delta 7.65(\mathrm{~d} \mathrm{2H}, \mathrm{Ar}) \\
& \delta 7.9(\mathrm{~d} 1 \mathrm{H}, \mathrm{Ar})\end{aligned}$ \\
\hline 11 & 4-OH acetophenone & 3-Benzoyl 4-OH acetophenone & 240 & $\begin{array}{c}\delta 2.9\left(\mathrm{~s} 3 \mathrm{H}, \mathrm{CH}_{3}\right) ; \delta 7.3(\mathrm{~m} 3 \mathrm{H}, \mathrm{Ar}) ; \delta 7.6(\mathrm{~d} 2 \mathrm{H}, \mathrm{Ar}) \\
\delta 8.45(\mathrm{~m} 2 \mathrm{H}, \mathrm{Ar}) ; \delta 8.8(\mathrm{~s} 1 \mathrm{H}, \mathrm{Ar}) ; \delta 10.9(\mathrm{~s} 1 \mathrm{H}, \mathrm{Ar}-\mathrm{OH})\end{array}$ \\
\hline 12 & 4-Me acetophenone & 3-Benzoyl 4-Me acetophenone & 238 & $\begin{array}{c}\delta 2.45\left(\mathrm{~s} 3 \mathrm{H}, \mathrm{CH}_{3}\right) ; \delta 2.85\left(\mathrm{~s} 3 \mathrm{H}, \mathrm{CH}_{3}-\mathrm{C}=\mathrm{O}\right) ; \delta 7.5(\mathrm{~m} \mathrm{3H}, \mathrm{Ar}) \\
\delta 7.7(\mathrm{~d} 2 \mathrm{H}, \mathrm{Ar}) ; \delta 8.5(\mathrm{~m} 2 \mathrm{H}, \mathrm{Ar}) ; \delta 8.9(\mathrm{~s} 1 \mathrm{H}, \mathrm{Ar})\end{array}$ \\
\hline 13 & 3-OH acetophenone & 3-Benzoyl 3-OH acetophenone & 240 & $\begin{array}{c}\delta 2.8\left(\mathrm{~s} 3 \mathrm{H}, \mathrm{CH}_{3}\right) ; \delta 7.45(\mathrm{~s} 1 \mathrm{H}, \mathrm{Ar}) ; \delta 7.6-7.8(\mathrm{~m} \mathrm{5H}, \mathrm{Ar}) \\
\delta 7.95(\mathrm{~s} 2 \mathrm{H}, \mathrm{Ar}) ; \delta 10.05(\mathrm{~s} 1 \mathrm{H}, \mathrm{Ar})\end{array}$ \\
\hline 14 & 4-Br acetophenone & 3-Benzoyl 4-Br acetophenone & 302 & $\begin{array}{c}\delta 2.65\left(\mathrm{~s} 3 \mathrm{H}, \mathrm{CH}_{3}\right) ; \delta 7.4(\mathrm{~m} 3 \mathrm{H}, \mathrm{Ar}) ; \delta 7.7(\mathrm{~d} 2 \mathrm{H}, \mathrm{Ar}) \\
\delta 8.5(\mathrm{~m} 2 \mathrm{H}, \mathrm{Ar}) ; \delta 8.7(\mathrm{~s} 1 \mathrm{H}, \mathrm{Ar})\end{array}$ \\
\hline 15 & 4- $\mathrm{NO}_{2}$ acetophenone & 3-Benzoyl $-\mathrm{NO}_{2}$ acetophenone & 269 & $\begin{array}{c}\delta 2.9\left(\mathrm{~s} 3 \mathrm{H}, \mathrm{CH}_{3}\right) ; \delta 7.3(\mathrm{~m} 3 \mathrm{H}, \mathrm{Ar}) ; \delta 7.6(\mathrm{~d} 2 \mathrm{H}, \mathrm{Ar}) \\
\delta 8.45(\mathrm{~m} 2 \mathrm{H}, \mathrm{Ar}) ; \delta 8.8(\mathrm{~s} 1 \mathrm{H}, \mathrm{Ar})\end{array}$ \\
\hline
\end{tabular}

\section{Conclusions}

In conclusion Vilsmeier-Haack Reagents [Acetamide and Oxychloride $\left(\mathrm{SOCl}_{2}\right.$ or $\left.\left.\mathrm{POCl}_{3}\right)\right]$ with aromatic aldehydes, ketones afforded acetyl derivatives under conventional (thermal) and non conventional [microwave irradiated (MIR), ultrasonic assisted and solvent free mortar pestle (grinding)] conditions with a trend: MIR (few seconds) $>>$ Sonication (minutes) $>$ Grinding $(\min )>>$ thermal (several hrs). Even though both the VH reagents afforded good to excellent yields of products reaction times were fairly less in the case of [Acetamide/ $\left./ \mathrm{POCl}_{3}\right]$ than those of $\left[\right.$ Acetamide $/ \mathrm{SOCl}_{2}$ ] reagent.

\section{Acknowledgements}

The authors thank CSIR, New Delhi for financial support in the form of Emeritus Scientist grant awarded to Prof. P. K. Saiprakash.

\section{References}

[1] T. W. Greene and P. G. M Wuts, "Protection Groups in Organic Synthesis," 3rd Edition, John Wiley, New York, 1999.

[2] G. Sartori, R. Ballini, F. Bigi, G. Bosica, R. Maggi and P. Righi, "Protection (and Deprotection) of Functional Groups in Organic Synthesis by Heterogeneous Catalysis," Chemical Reviews, Vol. 104, No. 1, 2004, pp. 199-250.

[3] A. L. Pearson and W. J. Roush, "Handbook of Reagents for Organic Synthesis; Activating Agents and Protecting Groups," John Wiley, Chichester, 1999.

[4] D. Harton, "Organic Synthesis Collective," Wiley, New York, 1991. 
[5] R. L. Zhdarov and S. M. Zherodarova, "Chemical Methods of Oligonucleotide Synthesis," Synthesis, Vol. 1975, No. 4, 1975, pp. 222-245. doi:10.1055/s-1975-23714

[6] G. Hoefle and W. Steglich, "N,N-Dimethyl-4-pyridinamine, a Very Effective Acylation Catalyst," Angewandte Chemie International Edition in English, Vol. 8, No. 12, 1969, p. 981. doi:10.1002/anie.196909811

[7] G. Hoefle, W. Steglich and H. Vorbrueggen, "4-Dialkylaminopyridines as Highly Active Acylation Catalysts. New Synthetic Method (25)," Angewandte Chemie International Edition in English, Vol. 17, No. 8, 1978, pp. 569-583.

[8] E. F. V. Scriven, “4-Dialkylaminopyridines: Super Acylation and Alkylation Catalysts," Chemical Society Reviews, Vol. 12, No. 2, 1983, pp. 129-161. doi:10.1039/cs9831200129

[9] T. Sano, K. Ohaschi and T. Oreyama, "Remarkably Fast Acylation of Alcohols with Benzoyl Chloride Promoted by TMEDA," Synthesis, Vol. 7, 1999, pp. 1141-1144.

[10] E. Vedejs, N. S. Bennett, L. M. L. Conn, S. T. Diver, M. Gingras, S. Lin, P. A. Oliver and A. L. Peterson, "Tributylphosphine-Catalyzed Acylations of Alcohols: Scope and Related Reactions," Journal of Organic Chemistry, Vol. 58, No. 25, 1993, pp. 7286-7288.

[11] E. Vedejs and S. T. Diver, "Tributylphosphine: A Remarkable Acylation Catalyst," Journal of the American Chemical Society, Vol. 115, No. 8, 1993, pp. 3358-3359. doi:10.1021/ja00061a056

[12] E. Vedejs, O. Daugulis and S. T. Diver, "Enantioselective Acylations Catalyzed by Chiral Phosphines," Journal of Organic Chemistry, Vol. 61, No. 2, 1996, pp. 430-431. doi:10.1021/j0951661v

[13] A. C. Cope and E. C. Herrich, "Organic Synthesis Collective," Wiley, New York, 1963.

[14] S. Chandrasekhar, T. R. Chander and M.Takhi, "Acylation of Alcohols with Acetic Anhydride Catalyzed by TaCl5: Some Implications in Kinetic Resolution," Tetrahedron Letters, Vol. 39, No. 20, 1998, pp. 3263-3266. doi:10.1016/S0040-4039(98)00465-1

[15] R. Dalpozzo, A. De Nino, L. Maiuolo, P. A. Procopio, M. Nardi, G. Bartoli and R. Romeo, "Highly Efficient and Versatile Acetylation of Alcohols Catalyzed by Cerium(III) Triflate," Tetrahedron Letters, Vol. 44, No. 30, 2003, pp. 5621-5624. doi:10.1016/S0040-4039(03)01358-3

[16] M. Moghadam, S. Tangestaninejad, V. Mirkhani, I. M. Baltrork and R. Shabani, "Rapid and Efficient Acetylation of Alcohols and Phenols with Acetic Anhydride Catalyzed by Electron-Deficient Tin(IV) Porphyrin," Journal of Moecular Catalysis A: Chemical, Vol. 219, No. 1, 2004, pp. 73-78. doi:10.1016/j.molcata.2004.05.004

[17] K. Ishihara, M. Kubota, H. Kurihara and H. Yamamoto, "Scandium Trifluoromethanesulfonate as an Extremely Active Lewis Acid Catalyst in Acylation of Alcohols with Acid Anhydrides and Mixed Anhydrides," Journal of Organic Chemistry, Vol. 61, No. 14, 1996, pp. 45604567. doi:10.1021/j0952237x
[18] P. A. Procopiou, S. P. D. Baugh, S. S. Flack and G. G. A. Inglis, "An Extremely Powerful Acylation Reaction of Alcohols with Acid Anhydrides Catalyzed by Trimethylsilyl Trifluoromethanesulfonate," Journal of Organic Chemistry, Vol. 63, No. 7, Vol. 63, 1998, pp. 2342-2347. doi:10.1021/jo980011z

[19] K. K. Chauhan, C. G. Frost, I. Love and D. Waite, "Indium Triflate, an Efficient Catalyst for Acylation Reactions," Synlett, Vol. 11, 1999, pp. 1743-1744. doi:10.1055/s-1999-2941

[20] D. Chandra, P. Saravanan, R. K. Singh and V. K. Singh, "Lewis Acid Catalyzed Acylation Reactions: Scope and Limitations," Tetrahedron, Vol. 58, No. 7, 2002, pp. 13691374. doi:10.1016/S0040-4020(01)01229-7

[21] A. Orita, G. Tañáis and A. Kakuda, "Highly Efficient and Versatile Acylation of Alcohols with $\mathrm{Bi}(\mathrm{OTf})_{3}$ as Catalyst," Angewandte Chemie International Edition, Vol. 39, No. 16, 2000, pp. 2877-2879. doi:10.1002/1521-3773(20000818)39:16<2877::AID-AN IE2877>3.0.CO;2-V

[22] M. L. Kantam, K. Aziz and P. R. Likhar, "Bis(Cyclopentadienyl) Zirconium Dichloride Catalyzed Acetylation of Phenols, Alcohols and Amines," Catalysis Communications, Vol. 7, No. 7, 2006, pp. 484-487. doi:10.1016/j.catcom.2005.10.001

[23] J. W. J. Bosco, A. Agrahari and A. K. Saikia, "Molecular Iodine Catalyzed Selective Acetylation of Alcohols with Vinyl Acetate," Tetrahedron Letters, Vol. 47, No. 24, 2006, pp. 4065-4068.

[24] M. A. Zolfigol, A. Khazaei, A. G. Choghamarani, A. Rostami and M. Hajjami, "Acylation of Alcohols Catalyzed by Using 1,3-Dibromo-5,5-dimethylhydentoin or Trichloroisocyanuric Acid," Catalysis Communications, Vol. 7, No. 6, 2006, pp. 399-402. doi:10.1016/i.catcom.2005.12.004

[25] H. T. Clarke and E. Rahrs, "Organic Syntheses. Collective Volumes," Coll, Vol. 1, 1941, p. 91

[26] J. Stawinski, T. Hozumi and S. A. Narang, "Benzoyltetrazole: A Mild Benzoylating Reagent for Nucleosides," Journal of the Chemical Society, Chemical Communications, Vol. 3, 1976, pp. 243-244. doi:10.1039/c39760000243

[27] M. Yamada, Y. Watabe, T. Sakakibara and R. Sudoh, "Preparation of a Water-Soluble Acylating Agent: Benzoylation of Acids, Amines, and Phenols with 2-Benzoylthio-1-methylpyridinium Chloride in Aqueous Phase,' Journal of the Chemical Society, Chemical Communications, Vol. 4, 1979, pp. 179-180. doi:10.1039/c39790000179

[28] F. A. Carey and K.O. Hodgson, "Efficient Syntheses of Methyl 2-O-Benzoyl-4,6-O-benzylidene- $\alpha$-D-glucopyrano-side and Methyl 2-O-Benzoyl-4,6-O-benzylidene- $\alpha-\mathrm{D}-$ ribo-hexopyranosid-3-ulose," Carbohydrate Research, Vol. 12 , No. 3, 1970, pp. 463-465. doi:10.1016/S0008-6215(00)80628-X

[29] T. W. Greene, "Protective Groups in Organic Synthesis," Wiley, New York, 1981. 
[30] C. B. Reese, "Protective Groups in Organic Chemistry," Plenum, London, 1973.

[31] S. Paul, P. Nanda and R. Gupta, "PhCOCl-Py/Basic Alumina as a Versatile Reagent for Benzoylation in Solvent-Free Conditions," Molecules, Vol. 8, 2003, pp. 374380. doi: $10.3390 / 80400374$

[32] G. A. Olah, St. J. Kuhn, "Friedls Craft's and Related Reactions," John Wiley and Sons, New York, 1964.

[33] H. Ulrich, "The Chemistry of Imidoyl Halides," Plenum Press, New York, 1968.

[34] R. Bonnet, "The Chemistry of the Carbon Nitrogen Double Bond," John Wiley Sons, New York, 1970. doi:10.1002/9780470771204.ch13

[35] E. Compaigne and W. L. Archer, "Organic Synthesis," John Wiley and Sons, New York, 1953.

[36] G. F. Smith, "Indoles. Part I. The Formylation of Indole and Some Reactions of 3-Formylindole," Journal of the Chemical Society, Vol. 1, 1954, pp. 3842-3846. doi:10.1039/jr9540003842

[37] R. N. Silverstein, C. Ryskiewez, C. Willert and R. C. Kocher, "Improved Synthesis of 2-Pyrrolealdehyde and of N-Methyl-2-Pyrrolealdehyde. Further Studies of Pyrrole Alcohols," Journal of Organic. Chemistry, Vol. 20, No. 5, 1955, pp. 668-672. doi:10.1021/jo01123a019

[38] A. Lorenz and R. Winzinger, "Über die Vinylenhomologen der Triphenylmethanfarbstoffe II," Helvetica Chimica Acta, Vol. 28, No. 1, 1945, pp. 600-612. doi:10.1002/hlca.660280183

[39] H. H. Boshard and H. Zollinger, "Die Synthese von Aldehyden und Ketonen mit Amidchloriden und Vilsmeier-Reagenzien," Helvetica Chimica Acta, Vol. 42, No. 5, 1959, pp. 1659-1671.

[40] S. Alumi, P. Linda, G. Marino, S. Santine and G. Salvelli, "The Mechanism of the Vilsmeier-Haack Reaction. Part II. A Kinetic Study of the Formylation of Thiophen Derivatives with Dimethylformamide and Phosphorus Oxychloride or Carbonyl Chloride in 1,2-Dichloroethane," Journal of the Chemical Society, Perkin Transactions, Vol. 2, No. 14, 1972, pp. 2070-2073.

[41] P. Linda, A. Lucccarelli, G. Marino and G. Savelli, "The Mechanism of the Vilsmeier-Haack Reaction. Part III.
Structural and Solvent Effects," Journal of the Chemical Society, Perkin Transactions, Vol. 2, No. 13, 1974, pp. 1610-1612.

[42] Z. Arnold and A. Holy, "Collection Czech," Chemical Communications, Vol. 27, 1962, pp. 2886-2895.

[43] T. D. Smith, "The Reaction of NN-Dimethylformamide with Phosphorus Trichloride," Journal of the Chemical Society A: Inorganic, Physical, Theoretical, 1966, pp. 841-842.

[44] G. J. Martin, S. Poignant, M. L. Filleux and M. T. Quemeneeuer, "Recherches sur la Reaction de VilsmeierHaack etude du Mecanisme de Formation du Complexe par des Mesures Cinetiques en Resonance Magnetique Nucleaire," Tetrahedron Letters, Vol. 11, No. 58, 1970, pp. 5061-5064.

[45] G. J. Martin and S. Poignant, "Nuclear Magnetic Resonance Investigations of Carbonium Ion Intermediates. Part I. Kinetics and Mechanism of Formation of the Vilsmeier-Haack Reagent," Journal of the Chemical Society, Perkin Transactions, Vol. 2, 1972, pp. 1964-1966. doi:10.1016/S0040-4039(00)96986-7

[46] K. C. Rajanna, F. Soloman, M. M. Ali and P. K. Saiprakash, "Vilsmeier-Haack Formylation of Coumarin Derivatives. A Solvent Dependent Kinetic Study," International Journal of Chemical Kinetics, Vol. 28, No. 12, 1996, pp. 865-875.

doi:10.1002/(SICI)1097-4601(1996)28:12<865::AID-KI $\mathrm{N} 1>3.0 . \mathrm{CO} ; 2-\mathrm{L}$

[47] J. G. Dingwall, D. H. Reid and K. Wade, "Studies of Heterocyclic Compounds. Part VI. NN-Dimethylth- ioform-Amide: A New Reagent in the Vilsmeier Reaction," Journal of the Chemical Society C: Organic, Vol. 6, 1969, pp. 913-915.

[48] T. L. Davis and W. E. Yelland, "Addition of Butylamine to Butyl Isocyanide," Journal of the American Chemical Society, Vol. 59, No. 10, 1937, pp. 1998-1999. doi:10.1021/ja01289a059

[49] A. Cipiciani, S. Clementi, P. Linda, G. Marino and G. Savelli, "Kinetics and Mechanism of N-Substitution of Indoles and Carbazoles in Vilsmeier-Haack Acetylation," Journal of the Chemical Society, Perkin Transactions, Vol. 2, 1977, pp. 1284-1287. 